\title{
A New Formalism for Studying Three-Body Interactions
}

\author{
Rosemary A. Mardling \\ School of Mathematical Sciences, Monash University, Melbourne, \\ Australia
}

\begin{abstract}
A new formalism for studying three-body interactions is discussed. It introduces the concept of forced modes of oscillation of a binary, and relies on the chaos theory concept of resonance overlap. The treatment provides a powerful tool for studying stability and scattering in hierarchical systems, and is not restricted by mass ratios, eccentricities or orientations.
\end{abstract}

\section{Introduction}

Almost all areas of astrophysics involve some aspect of the three-body problem. At the very least we can associate it with every area discussed at this symposium, and since the latter covered all length scales in astrophysics, it is clear that an understanding of three-body interactions in fundamental. Starting from the smallest scales (and revealing my interest in planetary dynamics), the following processes involve three-body interactions, and in particular illustrate that stability and scattering processes are most important: Bound-orbit scattering processes responsible for the formation of planet cores; migration of systems of newly formed planets as they interact with each other and the protoplanetary disk (the latter providing sources and sinks of energy and angular momentum); the stability of planetary systems once the disk is cleared; resonant capture processes which result in systems such as the $2: 1$ resonant pair of planets orbiting the star GJ 876; the stability of triple and higher-order star systems; scattering processes in star clusters including binary-single star interactions and binary-binary interactions, the latter including scattering, capture, and collisions resulting in the formation of new triples; scattering and collision processes in star-forming clouds; scattering and collision processes in clusters of galaxies.

Most astrophysical applications of the three-body problem have relied on studies of the planar circular restricted problem where one of the bodies is assumed to have negligible mass, although perturbation analyses involving small eccentricities and inclinations have been successfully applied to Solar System problems (Murray \& Dermott, 1999). Studies of stability of hierarchical triples have focussed mainly on what is referred to as "Hill stability" which uses energy and angular momentum considerations to predict which configurations are capable of suffering close encounters. It seems to be widely assumed that the escape of a body from a hierarchical triple is necessarily preceded by a close encounter, however this is definitely not the case! It is possible for the eccentricity of the outer orbit of a such a triple to random walk its way out past unity, thereby 
disrupting the configuration without a close encounter. This is related to a more general concept called "Lagrange stability", and a Lagrange stable system is one which remains bound for all time. While it is still impossible to predict which configurations are stable in this sense, the formulation discussed here allows one to predict which systems are dynamically Lagrange unstable in the sense that they are short-term unstable, irrespective of whether or not they are "allowed" to have close encounters. It may be used to study any hierarchical triple system and is not restricted by the mass ratios, eccentricities or orientations.

In addition to stability, the formalism may be used to study any one of the problems listed above including resonance capture, and in particular, is proving to be a powerful analytical tool for the study of scattering processes (Mardling, in preparation).

Section 2 reviews some general physical and mathemathical concepts, while section 3 introduces the concept of the forced modes of oscillation of a binary and presents some results which illustrate the success of the treatment. Section 4 presents a summary.

\section{General Concepts}

The new formalism relies on the concept of gravitational resonance and uses Hamiltonian mechanics as its mathematical basis. In particular, it utilizes a series of ideas beginning with the fundamental work of Poincaré on perturbed integrable systems (Poincarè 1993) and culminating in a paper by Wisdom (1981) in which stability in the restricted three-body problem is studied. The present work adds a crucial new concept to this elegant body of work, so that hierarchical triple systems (including unbound systems) can be studied in all their generality. Thus there is no restriction on the masses, the inner and outer eccentricities, or the relative orientation of the inner and outer orbits. The system should, however, have a clear hierarchical structure initially, although the study of subsequent exchange is possible.

A review of the mathematical ideas on which the present work relies may be found in Mardling (2001), while the complete theory will be published elsewhere. The following contains a summary.

\subsection{Gravitational Resonance}

Most people are used to thinking of what one might call linear resonance, that is, the kind of resonance which occurs if a linear oscillator is forced at its natural frequency. Resonance is associated with a large transfer of energy, and occurs because the forcing conditions are such that energy tends to flow in the same direction each forcing cycle. Now consider a stable system of two planets whose orbital periods are in the ratio $2: 1$, and imagine that their periastra are aligned and their conjunction point is near periastron ${ }^{1}$ (Figure 1(a), (b)(i)). Because the planets are in conjuction at around the same place in their orbits each cycle, there tends to be a net flow of energy to (or from) the outer orbit from (or to) the inner

\footnotetext{
${ }^{1}$ In fact, stable 2:1 resonant planetary systems tend to align their periastra or have them $180^{\circ}$ apart.
} 


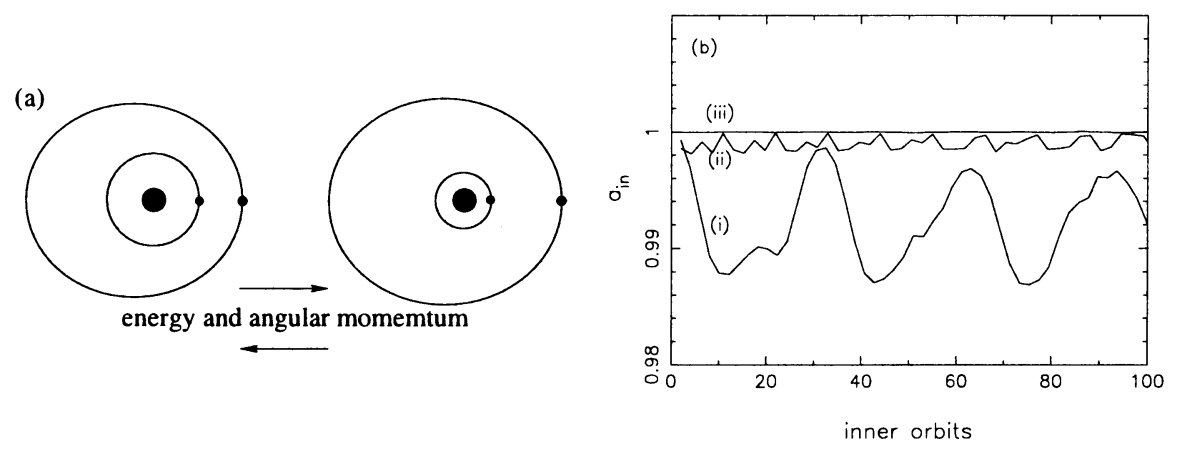

Figure 1. (a): A 2:1 planetary resonance with conjunction near periastron. (b)(i): A 2:1 resonance with masses $m_{1}=1, m_{2}=m_{3}=0.001$, a circular inner orbit and outer eccentricity 0.1 . (ii): Same as (i) but with the outer semi-major axis increased by $7 \%$. (iii): Same as (i) but with the outer semi-major axis doubled. Note that high frequency oscillations are not shown.

orbit. This is in contrast to non-resonant systems for which the orbital energies (and hence semi-major axes) remain secularly constant (Figure 1(b)(iii)). This flow of energy will, however, move the system away from resonance, reducing the amount per outer orbit until the direction of flow reverses and the system moves back towards resonance, and the conjunction points move back towards periastron. Thus the semi-major axes vary secularly, with the period of variation depending on how far the system is from exact resonance.

For a $2: 1$ resonance, the quantity $2 n_{\text {out }}-n_{\text {in }} \simeq 0$, where $n_{\text {in }}$ and $n_{\text {out }}$ are the inner and outer orbital frequencies (mean motions) respectively. The corresponding quantity $\phi_{21} \equiv 2 \lambda_{\text {out }}-\lambda_{\text {in }}$ then varies slowly, where $\lambda_{\text {in }}=\int n_{\text {in }} d t$ and similarly for $\lambda_{\text {out }}$. Since the kinds of problems for which the treatment is useful (dynamical stability and scattering) involve short timescales, it is assumed that secular motions such as apsidal motion, precession and nutation are negligible. The angle $\phi_{21}$ is called a resonance angle, and plays a fundamental role in the study of stability. For a resonant system, $\phi_{21}$ librates around zero, while for a system far from resonance, $\phi_{21}$ circulates. These concepts are familiar from the phase-space plot of the motion of a pendulum as Figure 2 illustrates. The width of a linear resonance is usually defined to be something like the full-width half maximum, but one may equally choose some other definition. In the present case, however, the width of a resonance has a precise definition and is the extent of the librating region, that is, the maximum vertical distance between separatrices.

\subsection{Resonance Overlap}

Poincaré introduced his General Problem of Dynamics in 1892 which asked the question: given an integrable system, that is, a Hamiltonian system for which the number of integrals of the motion is equal to the number of degrees of freedom (the number of independent coordinates), by how much can one perturb such a system before integrability is lost. This represented the birth of chaos theory, his 


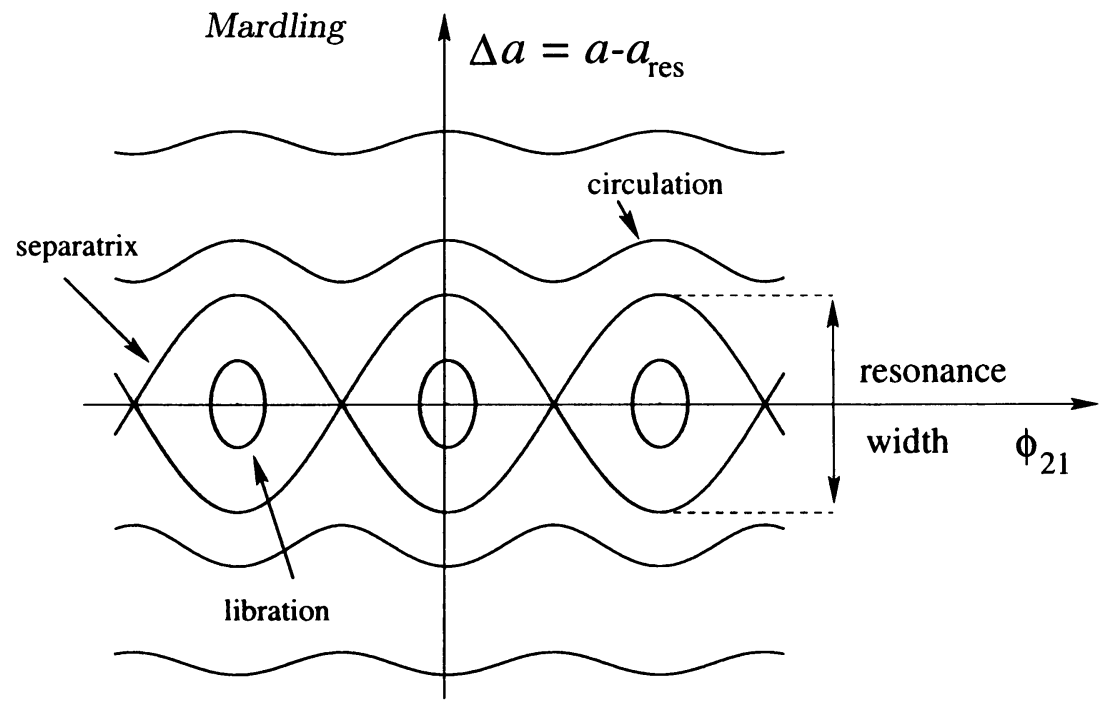

Figure 2. Variation of (either) semi-major axis with resonance angle $\phi_{21}=2 \lambda_{\text {out }}-\lambda_{\text {in }}$, illustrating libration and circulation. The amplitude of variation reduces away from resonance (see Figure 1). $a$ and $a_{\text {res }}$ are the inner or outer semi-major axis and the corresponding value at exact resonance respectively.

work inspired by the desire to win (pour la gloire de la France) a lucrative prize offered by King Oscar II of Sweden in 1885 (vive la concurrence!). He showed that a series solution to the (restricted) three-body problem could be expected to diverge because of small denominators caused by internal resonances. In 1954, Kolmogorov outlined a proof which was completed in the 1960s by Arnol'd and independently by Moser, which showed that such a series solution is convergent provided the natural frequencies associated with the orbital configuration are not "close" to resonance. ${ }^{2}$ This is the celebrated KAM theorem.

From a practical point of view, divergent series correspond to chaotic behaviour, and for the three-body problem this in turn corresponds to systems unstable to the escape of one body. But given its abstract form, it was noi clear how to use the KAM theorem for practical purposes until Chirikov (1979) proposed what we now refer to as the Chirikov resonance overlap criterion ${ }^{3}$. In particular, he studied the interaction of nonlinear oscillators and showed that one can quite accurately predict chaotic behaviour simply by calculating the width of individual resonances (in the "pendulum" sense of Figure 2) and then ask for what system parameters do neighbouring resonances "overlap". In reality, separatrices do not overlap, but rather exhibit extremely complex behaviour as they vie for the same phase space while not being allowed to cross (uniqueness). Nonetheless, this simple criterion provides a powerful method for determining the onset of chaos in Hamiltonian systems. In essence (and after a series of canonical transformations), the Hamiltonian is expanded around

\footnotetext{
${ }^{2}$ In fact their work was not restricted to the three-body problem.

${ }^{3}$ Which itself was inspired by the work of Walker and Ford (1969).
} 

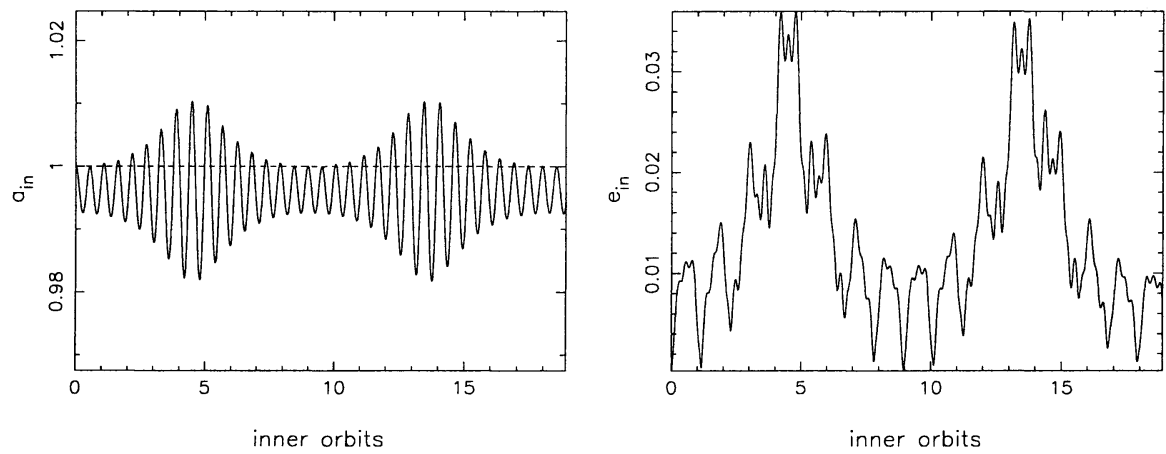

Figure 3. The forced oscillations of an equal mass binary. The inner binary is initially circular, and $a_{\text {out }}=4$ and $e_{\text {out }}=0.2$ initially. The amplitude increases during outer periastron passage.

the unperturbed state at resonance and in doing so, is cast in the form of the Hamiltonian for a pendulum. One can then calculate the width of a resonance using the equations for the separatrices and ask for what system parameters do neighbouring resonances overlap.

Finally Wisdom (1981) used the Chirikov resonance overlap criterion to study stability of the restricted three-body problem. It is his formulation upon which the present work is based.

\section{The Forced Modes of Oscillation of a Binary}

The concept introduced by the new formalism is that of forced modes of oscillation of a binary. Figure 3 shows the evolution of the inner semi-major axis, $a_{\mathrm{in}}$, and inner eccentricity, $e_{\text {in }}$, over two outer orbits for an equal-mass triple with $e_{\text {in }}=0.0, a_{\text {in }}=1, e_{\text {out }}=0.2$ and $a_{\text {out }}=4$ initially. The oscillation frequency of the inner semi-major axis is twice the inner orbital frequency, $n_{\text {in }}$, while the fundamental and third harmonics of the inner orbit dominate the inner eccentricity. These oscillations are fundamental to the stability of the system because it is these to which the outer orbit is coupled. It is useful to regard the inner orbit as an elliptic ring of material whose density decreases near periastron and increases near apastron, a device introduced by Gauss (at least for a static ring; see Murray \& Dermott 1999). Here, the ring oscillates as it exchanges energy with the outer orbit, at all times keeping the form of an ellipse (an "osculating" orbit). Note that this oscillatory motion is not associated with the interaction energy which is a separate part of the energy budget. Rather it is a part of the energy associated with the inner orbit.

The Hamiltonian for a hierarchical triple consisting of an inner binary with masses $m_{1}$ and $m_{2}$, and an outer body of mass $m_{3}$ can be written as

$$
H=H_{\text {in }}+H_{\text {out }}+H_{\text {int }},
$$

where

$$
H_{\text {in }}=\frac{1}{2} \mu_{\text {in }} \dot{\mathbf{r}}^{2}-\frac{G m_{1} m_{2}}{r}, \quad H_{\text {out }}=\frac{1}{2} \mu_{\text {out }} \dot{\mathbf{R}}^{2}-\frac{G m_{12} m_{3}}{R}
$$


and

$$
\begin{aligned}
H_{\mathrm{int}} & =\frac{G m_{12} m_{3}}{R}-\frac{G m_{1} m_{3}}{\left|\mathbf{R}+\alpha_{2} \mathbf{r}\right|}-\frac{G m_{2} m_{3}}{\left|\mathbf{R}-\alpha_{1} \mathbf{r}\right|} \\
& =-\frac{G \mu_{\mathrm{in}} m_{3}}{R} \sum_{l=2}^{\infty} \sum_{m=-l}^{l} \frac{4 \pi}{2 l+1} \mathcal{M}_{l}\left(\frac{r}{R}\right)^{l} Y_{l m}(\theta, \varphi) Y_{l m}^{*}(\Theta, \psi)
\end{aligned}
$$

describes the tidal interaction between the inner and outer orbits. Here $\mathbf{r}$ is the position vector of $m_{2}$ relative to $m_{1}$, and $\mathbf{R}$ is the position vector of $m_{3}$ relative to the centre of mass of the inner binary. These are called Jacobi coordinates. Parameters associated with the masses are $m_{12}=m_{1}+m_{2}, \mu_{\text {in }}=m_{1} m_{2} / m_{12}$, $\mu_{\text {out }}=m_{12} m_{3} / m_{123}, \alpha_{i}=m_{i} / m_{12}$ and $\mathcal{M}_{l}=\left(m_{1}^{l-1}+(-1)^{l} m_{2}^{l-1}\right) / m_{12}^{l-1}$. Note that $\mathcal{M}_{2}=1$ for any masses, while $\mathcal{M}_{l}=0$ when $l$ is odd and $m_{1}=m_{2}$. Thus, for example, the $l=3$ octupole term is important for planetary systems but not when the inner masses are similar. The sets of angles $(\theta, \varphi)$ and $(\Theta, \psi)$ are spherical polar coordinates associated with the vectors $\alpha_{1} \mathbf{r}$ and $\mathbf{R}$, both of which are measured from the centre of mass of the inner binary. The $Y_{l m}$ are spherical harmonics. Since secular motions are ignored, it is convenient to orientate the coordinate system such that $\varphi$ is the true anomaly of the inner orbit, and hence $\theta=\pi / 2$. For present purposes the spherical harmonic expansion (3) is truncated after the quadrupole $(l=2)$ term, however it is easy to generalize the formalism to include higher order terms.

The Hamiltonian for the inner binary is now written as the sum of two parts:

$$
H_{\text {in }}=H_{\text {in }}^{0}+H_{\mathrm{osc}}
$$

where $H_{\text {in }}^{0}=\frac{1}{2} \mu_{\text {in }}{\dot{\mathbf{r}_{0}}}^{2}-G m_{1} m_{2} / r_{0}$ is the Hamiltonian associated with an unperturbed inner binary $\left(m_{3}=0\right)$ with $\mathbf{r}_{0}$ the position vector of $m_{2}$ relative to $m_{1}$, and $H_{\mathrm{osc}}$ is the Hamiltonian associated with the oscillatory motion of a perturbed inner binary. Since $H_{\text {in }}^{0}$ is constant, it does not affect the dynamics and can be ignored. $H_{\text {osc }}$ is defined such that

$$
r=r_{0}+\eta \quad \text { and } \quad \dot{\varphi}=\dot{\varphi}_{0}+\zeta
$$

where

$$
\eta=\sum_{n=-\infty}^{\infty} b_{n}(t) e^{i n \varphi} \quad \text { and } \quad \zeta=\sum_{n=-\infty}^{\infty} a_{n}(t) e^{i n \varphi}
$$

and $r_{0}$ and $\dot{\varphi}_{0}$ are associated with the unperturbed inner binary. Since $\eta$ and $\zeta$ are real, $b_{-n}=b_{n}^{*}$ and $a_{-n}=a_{n}^{*}$. These expressions are consistent with regarding the inner binary as an oscillating Gauss ring, so that $e^{i n \varphi}$ is a forced mode of oscillation of such a ring and $b_{n}(t)$ is its amplitude. Note, however, that there are no free modes of oscillation as there are in the case of tidally forced modes of oscillation of a star. If the expressions (5) and (6) are substituted into the equations of motion of the (perturbed) inner binary and these equations are then expanded to first order in $\eta$ and $\zeta$, one can show that when the inner orbit is (initially) circular, $\eta$ satisfies the equation for forced simple harmonic motion. Equations for the complex amplitudes may be derived by taking advantage of the orthogonality of the mode functions $e^{i n \varphi}$. These equations are particularly 
simple for the case of a circular inner binary, otherwise they are also functions of $e_{\text {in }}$ (which may be regarded as constant).

Scattering processes can be studied analytically using the above normal mode analysis, together with a formulation similar to that of Press \& Teukolsky (1977) who studied stellar tidal capture. The following focuses on stability analysis and gives a brief overview of how the Chirikov resonance overlap criterion can be used to determine the stability of hierarchical triples.

Recall that the aim is to cast the Hamiltonian in the form of that for a pendulum, so that the widths of resonances may be calculated and hence their overlap. This is done in a series of steps. First the interaction term is truncated after the quadrupole term, expanded to first order in $\eta$ and integrated over the binary "ring". This produces an expression which governs the interaction between the modes of oscillation of the inner binary and the outer orbit. Subtracting the constant $H_{\text {in }}^{0}$, the new Hamiltonian, $\bar{H}$, becomes

$$
\bar{H}=H_{\text {osc }}+H_{\text {out }}+\bar{H}_{\text {int }} \equiv H_{0}+\bar{H}_{\text {int }}
$$

where $\bar{H}_{\text {int }}$ is the truncated (and smoothed) interaction term. $H_{0}$ is the integrable part of the Hamiltonian, consisting of a series of coupled linear oscillators and an uncoupled Kepler orbit, and $\bar{H}_{\text {int }}$ is the perturbation which may or may not destroy the integrability, depending on the configuration and hence whether or not neighbouring resonances "overlap". The first step towards a pendulum Hamiltonian is to write equation (7) in terms of action-angle variables. The two standard systems which many text books on classical mechanics use when they discuss action-angle variables are the harmonic oscillator and the Kepler orbit which are precisely the two systems used in this treatment. The interaction term is expanded in a Fourier series in these variables (and is simplified by the fact that secular frequencies are ignored). The next step is to ignore all Fourier terms except one resonant term which will be of the form $\cos \left(n \lambda_{\text {out }}-\vartheta_{m}\right) \equiv \cos \phi_{n m}$, where $\vartheta_{m}=m \lambda_{\text {in }}$ is the angle variable associated with the resonant oscillator of interest. The angle $n \lambda_{\text {out }}$ is associated with the nth harmonic of the outer orbit, the strength of which increases with increasing $e_{\text {out }}$, and similarly for $m \lambda_{\text {in }}$. The resonant angle $\phi_{n m}$ is associated with the $n: m$ resonance. Another canonical transformation to "resonance variables" is performed, and the resulting Hamiltonian is expanded in a Taylor series "about resonance", being truncated after the quadratic term leaving the Hamiltonian for a pendulum. Figure 4(a) shows how the widths of the $n: 1$ resonances depend on $e_{\text {out }}$ by plotting $e_{\text {out }}$ against $r=n_{\text {in }} / n_{\text {out }}=T_{\text {out }} / T_{\text {in }}$, where $T_{\text {in }}$ and $T_{\text {out }}$ are the inner and outer orbital periods respectively. Exact resonance corresponds to integer values of $r$. The dashed line corresponds to the $2: 1$ resonance which is the only $n: 1$ resonance which has finite width for $e_{\text {out }}=0$. Unstable triples have values for $e_{\text {out }}$ and $r$ which are common to two or more resonances. Figure 4(b) shows the stability boundary for an equal-mass triple. The dots are determined numerically and correspond to unstable systems (see Mardling (2001) for a discussion of how these are obtained); the circles correspond to where neighbouring resonances first overlap; the solid curve comes from the semi-analytical formula $a_{\text {out }} / a_{\text {in }}=C r^{4}$, where $C \simeq 0.013$ for circular inner binaries with $m_{1}=m_{2}$ (again, see Mardling (2001)). 

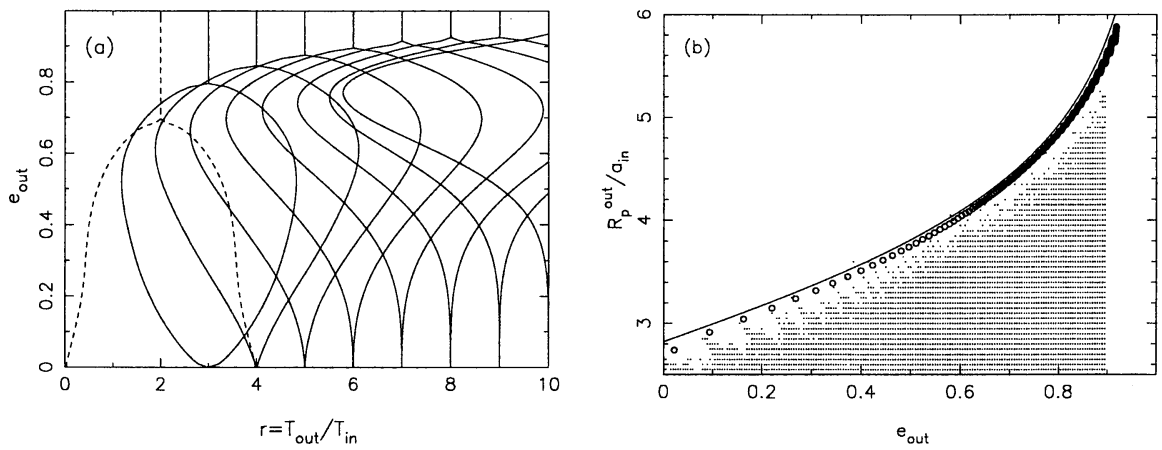

Figure 4. (a): The overlap of neighbouring $n: 1$ resonances for a hierarchical triple with a circular inner binary and $m_{1}=m_{2}=1, m_{3}=$ 0.05. (b): The stability boundary for an equal-mass triple with $e_{\text {in }}=0$. Dots indicate unstable systems and are determined numerically; circles are theoretical predictions; the solid curve is semi-empirical. $R_{p}^{\text {out }}$ is the outer periastron distance.

\section{Summary}

The new formalism which introduces the concept of the forced modes of oscillation of a binary and uses the theory of resonance overlap is clearly successful at predicting the stability of similar-mass triples. It has been tested for a range of mass ratios and is equally successful for these, although some systems with small $e_{\text {out }}$ are sensitive to the omission of secular frequencies. All the information about masses, eccentricities and orientation appears in the interaction term (see equation (3)), and so the theory can in principle be used for any hierarchical system. For practical applications such as triple formation in star cluster simulations, the formula in the previous paragraph is used, with $C$ depending on other parameters (Mardling \& Aarseth, 2001). Use of the normal mode analysis introduced here is also proving to be a powerful tool for studying scattering processes (Mardling, in preparation).

\section{References}

Chirikov, B. V. 1979, Phys. Rep., 52, 263

Mardling, R. A. 2001, astro-ph and ASP Conference Series, Vol. 229, eds Podsiadlowski, P., Rappaport, S., King, A.R., D'Antona, F. \& Burderi, L.

Mardling, R. A. \& Aarseth, S. J. 2001, MNRAS, 321, 398

Murray, C. D. and Dermott, S. F. 1999, Solar System Dynamics, CUP, 293

Poincaré, H. 1993, New Methods of Celestial Mechanics (Vol 1), ed. D. L. Goroff (New York: AIP), I23, 22

Press, W. H. \& Teukolsky, S. A. 1977, ApJ, 213, 183

Walker, G. H. \& Ford, J. 1969, Phys. Rev. 188, 416

Wisdom, J. 1980, AJ, 85, 1122 